\title{
Electrocardiogram profiling of myocardial infarction history using MLP and HMLP networks
}

\author{
Fatin Syahirah Ab Gani ${ }^{1}$, Mohd Khairi Nordin², Ahmad Ihsan Mohd Yassin ${ }^{3}$, Idnin Pasya Ibrahim ${ }^{4}$, \\ Megat Syahirul Amin Megat $\mathrm{Ali}^{5}$ \\ ${ }^{1,2,3}$ Faculty of Electrical Engineering, Universiti Teknologi MARA, Malaysia \\ ${ }^{4,5}$ Microwave Research Institute, Universiti Teknologi MARA, Malaysia
}

\begin{tabular}{l}
\hline Article Info \\
\hline Article history: \\
Received Apr 30, 2019 \\
Revised Jul 5, 2019 \\
Accepted Jul 30, 2019 \\
\hline
\end{tabular}

\section{Keywords:}

Electrocardiogram

Hybrid multilayered perceptron

Multilayered perceptron

Myocardial infarction

Power ratio

\begin{abstract}
Narrowing of coronary arteries caused by cholesterol deposits deprives heart tissues of oxygen. In prolonged conditions, these will result in myocardium infarction. The presence of damage tissues modifies the normal sinus rhythm and this can be detected using electrocardiogram (ECG). Hence, this paper characterized history of myocardial infarction from survivors using QRS power ratio features from the ECG. Subsequent profiling is performed using multilayered perceptron (MLP) and hybrid multilayered perceptron (HMLP) networks. ECG with history of anterior and inferior infarctions, along with healthy controls is obtained from PTB Diagnostic ECG Database. The signal is initially pre-processed and the power ratio features are extracted for lowand mid-frequency components. The features are then used as input vector to the MLP and HMLP networks. The optimized MLP has attained accuracies of $99.2 \%$ for training and $98.0 \%$ for testing. Meanwhile, the optimized HMLP managed to achieve accuracies of $99.4 \%$ for training and $97.8 \%$ for testing. Despite the similarities in network performance, MLP provides a better alternative due to the reduced computational requirements by as much as $30 \%$.
\end{abstract}

Copyright $\odot 2020$ Institute of Advanced Engineering and Science. All rights reserved.

\section{Corresponding Author:}

Megat Syahirul Amin Megat Ali,

Microwave Research Institute,

Universiti Teknologi MARA,

40450 Shah Alam, Malaysia.

Email: megatsyahirul@uitm.edu.my

\section{INTRODUCTION}

Acute myocardial infarction is caused by prolonged ischemic conditions which often result in necrosis of myocardium tissues. Delay in delivery of treatment will lead to cardiac arrest and in most cases, death [1-2]. Previously, studies have established ECG pattern for patients who survived myocardial infarction. These were initially grounded on the notion that impaired heart tissues will induce abnormalities to the healthy sinus rhythm [3-4]. These can be detected through the ECG; a non-invasive technique for recording electrical activities of the heart. Such approach is made possible through the use of specialized silver-chloride electrodes that are connected to the limbs; effectively forming the leads that represent the frontal plane of the heart [5]. The lead orientations enable detection of abnormalities within the electrical conduction system; hence allowing a more targeted and precise treatment [6]. Apart from myocardial infarction, previously studied arrhythmias are cardiomyopathy [7-8], premature ventricular contractions [9-10], and bundle branch blocks [11-12].

Past studies involving profiling of myocardial infarction history have implemented the ECG power ratio features from both of the frontal limb leads. These were based on three pre-defined frequency components of the QRS-complex. The results however, indicate that only the low-frequency $(5-15 \mathrm{~Hz})$ and mid-frequency $(15-50 \mathrm{~Hz})$ zones are capable of discriminating healthy control from ECG with history of 
anterior and inferior infarctions with acceptable accuracies. A comparative analysis have also highlighted that the information from both bipolar and unipolar limb leads yielded similar performance [13]. Therefore, either one of the lead system would suffice for the development of more advanced profile model.

Previously, history profiling has been performed using MLP network. The intelligent model yielded satisfactory performance using the low- and mid-frequency QRS power ratio features [13]. MLP is advantageous as it is able at learning complex non-linear relationships and generalizes solution for a given problem [14-15]. In the past, the ECG profiling model was optimized using modified constructive algorithm which assesses optimum number of hidden nodes based on best average accuracies and mean squared error (MSE) [16]. The methods for setting the upper and lower boundary conditions are flawed as it is derived from limited assumptions. Often, a better alternative in terms of number of hidden nodes is attained by selecting values beyond the range of conventional boundary conditions [10].

Similar optimization issues are also present for HMLP network. The hybrid structure is an improvement of MLP; with parallel weighted connection directly from input and the output node. Studies have shown that the network is capable of modelling linear and non-linear relationships with better accuracies compared to the conventional MLP. For function approximation problems however, it has shown to consume more time for network training [17-18]. To date, HMLP has yet to be implemented to profile ECG with myocardial infarction history.

Hence, three objectives have been outlined in this study; 1) to characterize QRS power ratio features for healthy control, as well as ECG with history of anterior and inferior myocardial infarction, 2) to develop ECG profile models using MLP and HMLP network, and 3) to evaluate the best network model based on classification performance and computational requirements.

\section{RESEARCH METHOD}

The initial part of the study encompasses data acquisition, signal pre-processing, as well as extraction of low- (LF-QRS) and med-frequency (MF-QRS) power ratio features. Subjects are then segregated into classes of anterior and inferior infarction histories, as well as healthy controls. Subsequently, the structure of MLP and HMLP network is optimized using modified constructive algorithm. The best number of hidden nodes is implemented in the development of ECG profile models using both MLP and HMLP. Finally, performance of both network structures are analyzed in terms of accuracy and computational requirements.

\subsection{Data Collection and Signal Pre-Processing}

Comprehensive data with infarction histories, along with the healthy controls is acquired from the PTB Diagnostic ECG database. ECG has been recorded at sampling rate of $1 \mathrm{kHz}$ using the PhysikalischTechnische Bundesanstalt prototype device [19]. In this study, only bipolar limb lead is selected. Table 1 shows the number of subjects for each ECG class and its corresponding indexes.

Table 1. Number of Subjects for the Respective ECG Class

\begin{tabular}{ccc}
\hline ECG Class & Number of Subjects & Index \\
\hline Anterior infarction & 27 & 1 \\
Inferior infarction & 36 & 2 \\
Healthy control & 24 & 3 \\
\hline
\end{tabular}

\subsection{Extraction of QRS Power Ratio Features}

ECG is filtered into LF-QRS and MF-QRS components using finite impulse response band-pass filters [20]. The ECG is segregated into smaller segments of five second sample. Hence, the total number of samples obtained is 1206 each class. Each sample is transformed to power spectral density (PSD). The procedure is performed using Welch technique. Subsequently, the area under PSD curve is quantified as energy spectral density (ESD). The ESD is then normalized using (1), (2) and (3), where I, II and III each refers to information from Lead I, II and III [13]. The power ratio, computed for both frequency components are then clustered into the respective ECG classes for pattern analysis. These are performed using box plots in SPSS.

$$
\text { Power Ratio I }=\frac{\mathrm{ESD}_{\mathrm{I}}}{\mathrm{ESD}_{\mathrm{I}}+\mathrm{ESD}_{\mathrm{II}}+\mathrm{ESD}_{\mathrm{III}}}
$$




$$
\begin{aligned}
& \text { Power Ratio II }=\frac{\mathrm{ESD}_{\mathrm{II}}}{\mathrm{ESD}_{\mathrm{I}}+\mathrm{ESD}_{\mathrm{II}}+\mathrm{ESD}_{\mathrm{III}}} \\
& \text { Power Ratio III }=\frac{\mathrm{ESD}_{\mathrm{III}}}{\mathrm{ESD}_{\mathrm{I}}+\mathrm{ESD}_{\mathrm{II}}+\mathrm{ESD}_{\mathrm{III}}}
\end{aligned}
$$

\subsection{Multilayered Perceptron Network}

Generally, the MLP is composed of an input layer, several hidden layers and an output layer. Studies have shown that single hidden layer is sufficient for function approximation with acceptable level of accuracies. The number of input nodes depends on the size of feature vector. Therefore in this study, the network is fed by six inputs. Conversely, only one output node which corresponds to the ECG indexes is implemented. As expressed by (4), feature input, xi is converted to vector of hidden variables, uj via activation function, $\Gamma 1 . \mathrm{M}$ is the number of input nodes, wij is the weights between ith input to jth hidden node, and $\theta \mathrm{j}$ are the biases. $\Gamma 1$ adopts hyperbolic tangent function [21-22].

$$
u_{j}=\Gamma_{1}\left(\sum_{i=1}^{M} w_{i j} x_{i}+\theta_{j}\right)
$$

Consequently, vector uj is transformed into the resultant output, yk, via activation function $\Gamma 2$. This can be expressed by (5), where $\mathrm{N}$ is the number of hidden nodes, wjk is the weights between jth hidden node to $\mathrm{kth}$ output node, and $\theta \mathrm{k}$ are the biases. $\Gamma 2$ adopts the pure linear function to approximate the corresponding class indexes [21-22].

$$
y_{k}=\Gamma_{2}\left(\sum_{j=1}^{N} w_{j k} u_{j}+\theta_{k}\right)
$$

The error, computed as the difference between $\mathrm{yk}$ and the desired output is integrated into the Levenberg-Marquardt algorithm for network training [23-24]. Convergence of error is monitored via MSE. In this study, early-stopping criterion is implemented to avoid network from over-fitting. A separate validation data is used to intermittently evaluate the network for its generalization ability. Training is halted when validation error increases. Testing set is used to assess performance of the fully trained network. The dataset is randomly segregated for training, validation and testing with a ratio of 70:15:15.

The best number of hidden nodes is evaluated using modified constructive algorithm. For every hidden node configuration, the network is trained forty times. At each training cycle, the network will reset at randomized starting weights and biases. Hence, an optimum number of hidden nodes will induce the best average performance, irrespective of pseudorandom Mersenne twister settings. The method determines the most suitable number of hidden nodes through the highest average accuracy and lowest average MSE during training. In this study, the number of hidden nodes ranges from five to twenty.

\subsection{Hybrid Multilayered Perceptron Network}

HMLP is a modification of MLP architecture with additional connections from input to the output layer. With this structure, yk no longer rely only on vector uj, but also considers vector xi. This can be mathematically expressed by (6) [25-26].

$$
y_{k}=\Gamma_{2}\left(\sum_{j=1}^{N} w_{j k} u_{j}+\theta_{k}+\sum_{i=1}^{M} w_{i k} x_{i}\right)
$$

Due to the presence of linear component, the complexity of computational requirements increases by an additional six multiplier and adder operations. This will be reflected in longer processing time. Despite the limitations, HMLP has been widely implemented to model non-linear relationships and has shown to exhibit superior performance than the preceding MLP. In this study, development of ECG profile models using HMLP adopts similar configurations as MLP in terms of optimization method, activation functions, learning algorithm, early-stopping criterion, and dataset segregation for training, validation and testing. 


\subsection{Performance Metrics}

The parameters used to evaluate model performance are accuracy (Acc), sensitivity (Se) and positive predictivity (Pp). Each is expressed by (7), (8) and (9). TP is the true positive, TN is the true negative, FP is the false positive, and FN is the false negative classifications.

$$
\begin{aligned}
& A c c=\left(\frac{T P+T N}{T P+T N+F P+F N}\right) \times 100 \% \\
& S e=\left(\frac{T P}{T P+F P}\right) \times 100 \% \\
& P p=\left(\frac{T P}{T P+F N}\right) \times 100 \%
\end{aligned}
$$

\section{RESULTS AND DISCUSSION}

Initially, the discussion elaborates on the characteristics of QRS power ratio features for ECG with infarction histories, along with the healthy controls. This is followed by optimization and development of ECG profile model using MLP network. Subsequently, similar scope of discussion ensues for ECG profile model via HMLP network. Performance of both methods is compared based on overall accuracy and computational requirements.

\subsection{Characterization of QRS Power Ratio Features for Different ECG Profiles}

The pattern of mean and distribution of LF-QRS power ratio for the three control groups is shown in Figure 1. Healthy control exhibit the lowest mean for Lead I, followed by those with anterior and then, inferior infarctions. Meanwhile for Lead II, the lowest median is attained by subjects with damage to inferior, followed by anterior aspects of the heart. It is worth noting that the median for both groups is almost similar. Conversely, the lowest median for Lead III has been attained by healthy controls, followed by subjects who survived anterior and subsequently, inferior infarctions. No extreme outliers have been observed.

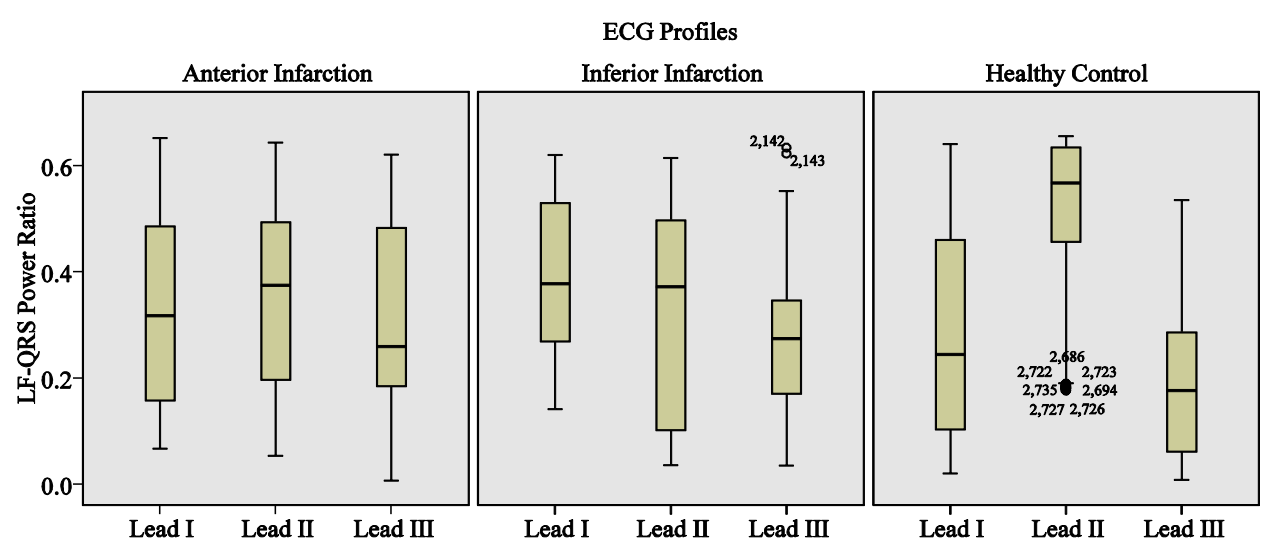

Figure 1. Pattern and distribution of LF-QRS power ratio for different ECG profiles

Consequently, Figure 2 shows the pattern of median and distribution of MF-QRS power ratio for the three control groups. The lowest median for Lead I is attained by healthy controls, followed by subjects who suffered from anterior and then, inferior infarctions. Meanwhile for Lead II, the lowest median is yielded by those with damage to anterior, followed by inferior positions. Healthy controls have attained the highest median for Lead II. Conversely, the lowest median for Lead III is obtained by healthy controls, followed by subjects with inferior and subsequently, anterior infarctions. No extreme outlier has been observed for MFQRS power ratio features. 


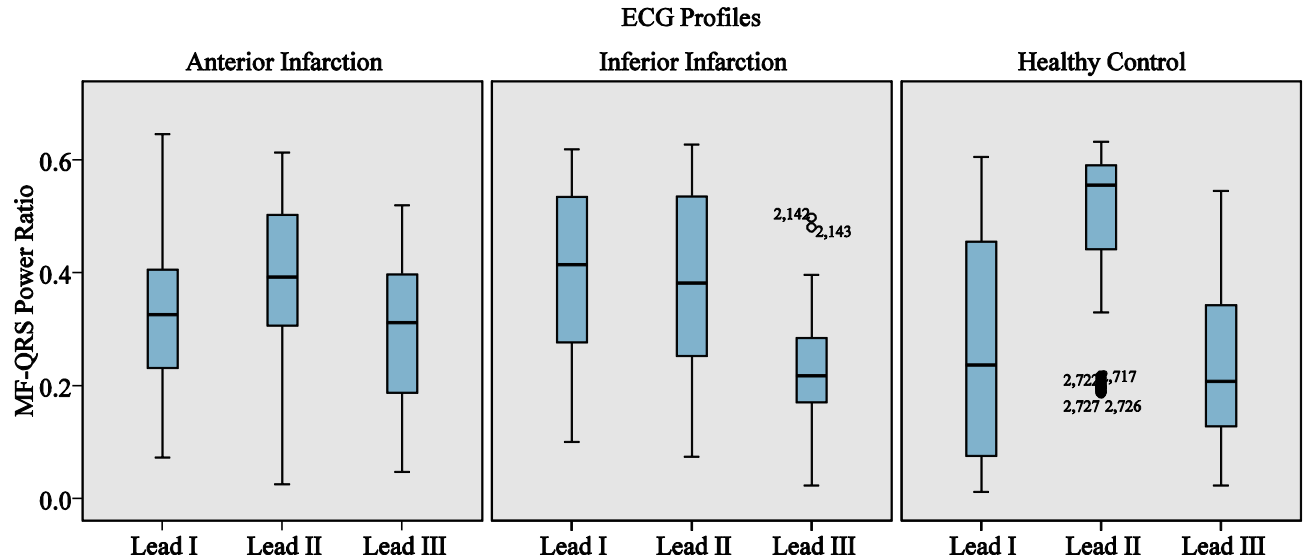

Figure 2. Pattern and distribution of MF-QRS power ratio for different ECG profiles

\subsection{Development of ECG Profile Model Using Multilayered Perceptron Network}

The average training accuracies and MSE for different hidden node configurations in MLP is shown in Figure 3. The average accuracies increase from $63.2 \%$ for five hidden nodes, to $99.3 \%$ for twenty hidden nodes. Meanwhile, the trend of MSE indicates a reciprocal relationship with the preceding results. The average MSE decreases from 0.23 for five hidden nodes, to 0.02 for twenty hidden nodes. To maintain balance between excellent model performance and increasingly complex computational requirement, the optimum number of hidden nodes is selected at thirteen with approximate accuracy of $95.0 \%$ and MSE of 0.05 for training.

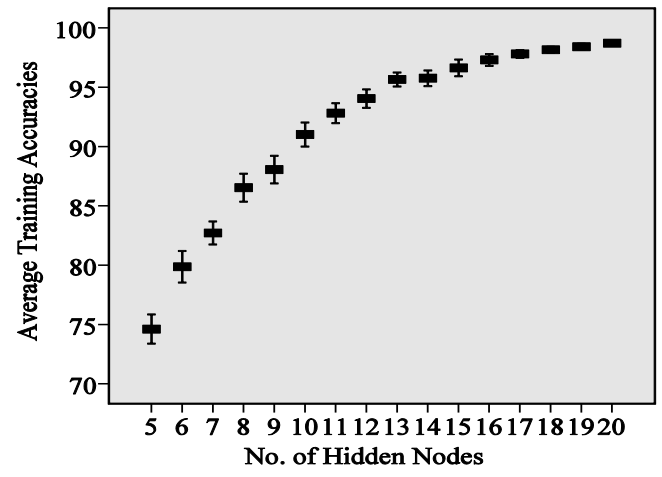

(a)

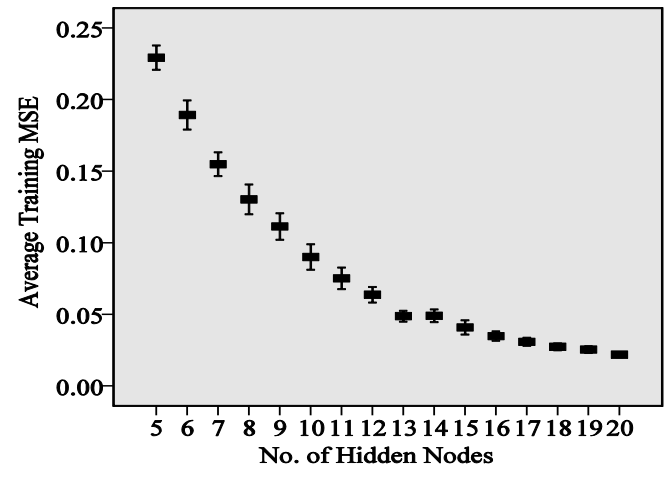

(b)

Figure 3. Average training (a) accuracies and (b) MSE analysis for MLP network

The ECG profile model is then developed with the optimized network structure. As shown in Table 2, excellent performance has been achieved with accuracies of $98.0 \%$ for training, $97.3 \%$ for validation and $99.2 \%$ for testing. Sensitivity and positive predictivity measures further indicate that despite the high degree of feature overlapping, the model is able to discriminate between control groups with exceptional accuracies.

Table 2. Model Performance using MLP Network

\begin{tabular}{|c|c|c|c|c|c|}
\hline \multicolumn{2}{|c|}{ Parameters } & Anterior Infarction & Inferior Infarction & Healthy Controls & $A c c$ \\
\hline \multirow{2}{*}{ Training } & $\mathrm{Se}$ & $98.4 \%$ & $97.5 \%$ & $96.2 \%$ & \multirow{2}{*}{$98.0 \%$} \\
\hline & $P p$ & $98.3 \%$ & $96.9 \%$ & $98.9 \%$ & \\
\hline \multirow{2}{*}{ Validation } & $\mathrm{Se}$ & $97.8 \%$ & $98.1 \%$ & $96.0 \%$ & \multirow{2}{*}{$97.3 \%$} \\
\hline & $P p$ & $98.9 \%$ & $93.5 \%$ & $99.4 \%$ & \\
\hline \multirow{2}{*}{ Testing } & $\mathrm{Se}$ & $99.4 \%$ & $98.8 \%$ & $99.4 \%$ & \multirow{2}{*}{$99.2 \%$} \\
\hline & $P p$ & $100 \%$ & $98.8 \%$ & $98.9 \%$ & \\
\hline
\end{tabular}




\subsection{Development of ECG Profile Model Using Hybrid Multilayered Perceptron Network}

For HMLP network, the average training accuracies and MSE for different hidden node configurations is shown in Figure 4. The average accuracies increase from $70.4 \%$ for five hidden nodes, to 99.5\% for twenty hidden nodes. At five hidden nodes, HMLP has already surpassed the performance of MLP network. The average training MSE for varying number of hidden nodes in HMLP is shown Figure 4. The pattern indicates a reciprocal relationship with the prior results. The average MSE decreases from 0.21 for five hidden nodes, to 0.02 for twenty hidden nodes. Similar to MLP, the optimum number of hidden nodes is selected at thirteen with approximate accuracy of $95.0 \%$ and MSE of 0.05 for training.

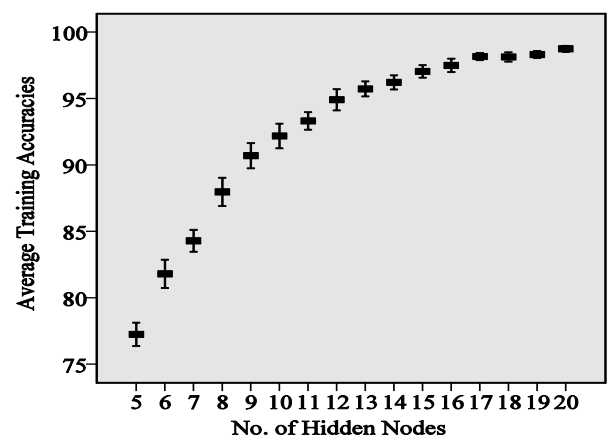

(a)

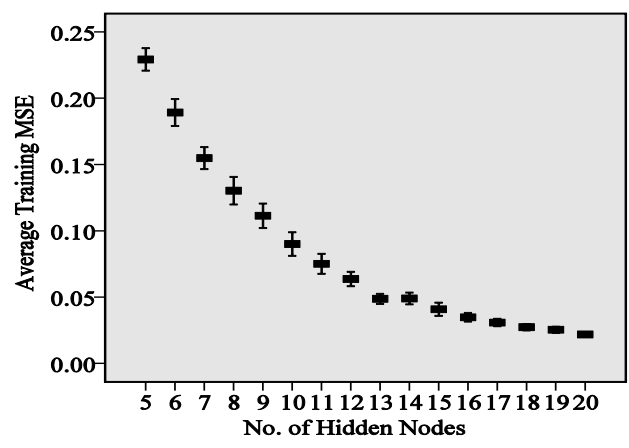

(b)

Figure 4. Average training (a) accuracies and (b) MSE analysis for HMLP network

The ECG profile model is then developed with the optimized network structure. As shown in Table 3, excellent performance has been attained with accuracies of $97.9 \%$ for training, $98.5 \%$ for validation and $99.4 \%$ for testing. Similarly with MLP, the model is still able to discriminate between subjects who survived anterior and inferior infarctions, as well as healthy controls with exceptional accuracies.

Table 3. Model Performance using HMLP Network

\begin{tabular}{|c|c|c|c|c|c|}
\hline \multicolumn{2}{|c|}{ Parameters } & Anterior Infarction & Inferior Infarction & Healthy Controls & $A c c$ \\
\hline \multirow{2}{*}{ Training } & $\mathrm{Se}$ & $99.1 \%$ & $96.3 \%$ & $98.5 \%$ & \multirow{2}{*}{$97.9 \%$} \\
\hline & $P p$ & $98.5 \%$ & $97.6 \%$ & $97.7 \%$ & \\
\hline \multirow{2}{*}{ Validation } & $\mathrm{Se}$ & $98.9 \%$ & $97.5 \%$ & $98.8 \%$ & \multirow{2}{*}{$98.5 \%$} \\
\hline & $P p$ & $99.5 \%$ & $97.5 \%$ & $98.2 \%$ & \\
\hline \multirow{2}{*}{ Testing } & $\mathrm{Se}$ & $100 \%$ & $99.5 \%$ & $98.8 \%$ & \multirow{2}{*}{$99.4 \%$} \\
\hline & $P p$ & $100 \%$ & $99.0 \%$ & $99.4 \%$ & \\
\hline
\end{tabular}

\subsection{Performance Comparison}

Thus far, ECG profile models have been successfully developed from QRS power ratio features using MLP and HMLP. Despite the structural differences, both networks are optimized at thirteen hidden nodes. Table 4 compares the number of multiplier and adder operators required for the proposed methods.

Table 4. Number of Operators for MLP and HMLP Networks

\begin{tabular}{cccc}
\hline Network & Layer & Multiplier & Adder \\
\hline \multirow{2}{*}{ MLP } & $u_{j}$ & 91 & 78 \\
& $y_{k}$ & 14 & 13 \\
\multirow{2}{*}{ HMLP } & $u_{j}$ & 91 & 78 \\
& $y_{k}$ & 20 & 19 \\
\hline
\end{tabular}

The number of operators remains unchanged at hidden layer. However, the effects of additional connection can be observed for the output node. Hence for forward propagating computation, the load for multiplier and adder operations increases by $42.9 \%$ and $46.2 \%$, respectively. Meanwhile, similarities have also been observed in terms of classification accuracies. Thus, MLP is recommended over HMLP as it is able to provide optimum performance, even in the absence of additional input-output connection. 


\section{CONCLUSION}

This paper presents an ECG profiling of myocardial infarction history using MLP and HMLP network. The three outlined objectives earlier have successfully been solved. Initially, ECG with infarction survivors and healthy controls are characterized using QRS power ratio features from bipolar limb leads. Subsequently, the proposed MLP and HMLP network structures have been optimized as ECG profile models with excellent classification accuracies. Despite the similarities in terms of performance, analysis shows that MLP is comparatively more efficient than HMLP as it reduces computational requirements by up to $30 \%$.

\section{ACKNOWLEDGEMENTS}

The study was funded by the Faculty of Electrical Engineering and Institute of Research Management and Innovation, Universiti Teknologi MARA (600-IRMI/MyRA 5/3/LESTARI (015/2017)).

\section{REFERENCES}

[1] K. Thygesen, et al., "Fourth Universal Definition of Myocardial Infarction," Journal of American College of Cardiology, vol. 72, pp. e618-0e651, 2018.

[2] L. Witten, et al., "Reasons for Death in Patients Successfully Resuscitated from Out-of-Hospital and In-Hospital Cardiac Arrest," Resuscitation, vol. 136, pp. 93-99, 2019.

[3] J. W. Waks and A. E. Buxton, "Risk Stratification for Sudden Cardiac Death After Myocardial Infarction," Annual Review of Medicine, vol. 69, pp. 147-164, 2018.

[4] M. S. A. Megat Ali, et al., "Characterization of Post-MI Electrocardiogram using Power Ratio Features and kNearest Neighbor Classifier," Journal of Fundamental and Applied Sciences, vol. 9, pp. 937-951, 2017.

[5] J. G. Webster, Medical Instrumentation: Application and Design, 4th ed. New Jersey: Wiley, 2009.

[6] L. Lancia, et al., "Continuous ECG Monitoring in Patients With Acute Coronary Syndrome or Heart Failure: EASI Versus Gold Standard," Clinical Nursing Research, vol. 27, pp. 433-449, 2018.

[7] A. Lyon, et al., "Distinct ECG Phenotypes Identified in Hypertrophic Cardiomyopathy using Machine Learning Associate With Arrhythmic Risk Markers," Frontiers in Physiology, vol. 9, 2018.

[8] M. H. Ahmad Shukri, et al., "Investigation on Elman Neural Network for Detection of Cardiomyopathy," in Proceedings of 2012 IEEE Control and System Graduate Research Colloquium, 2012, pp. 328-332.

[9] J. S. Lim, "Finding Features for Real-Time Premature Ventricular Contraction Detection Using a Fuzzy Neural Network System," IEEE Transactions on Neural Networks, vol. 20, pp. 522-527, 2009.

[10] A. Ebrahimzadeh and A. Khazaee, "Detection of Premature Ventricular Contractions using MLP Neural Networks: A Comparative Study,” Measurement, vol. 43, no. 1, pp. 103-112, 2010.

[11] B. Lin, et al., "A Single Lead of Concordant ST Deviation in Left Bundle Branch Block," Annals of Emergency Medicine, vol. 34, pp. 409-412, 2019.

[12] M. S. A. Megat Ali, et al., "Hybrid Multilayered Perceptron Network for Classification of Bundle Branch Blocks," in Proceedings of 2012 International Conference on Biomedical Engineering, 2012, pp. 149-154.

[13] A. H. Hussin, et al., "Profiling of Myocardial Infarction History from Electrocardiogram using Artificial Neural Network," International Journal of Engineering \& Technology, vol. 7, no. 4.11, pp. 236-240, 2018.

[14] W. Cao, et al., "A Review on Neural Networks with Random Weights," Neurocomputing, vol. 275, pp. 278-287, 2018.

[15] M. Negnevitsky, “Artificial Intelligence: A Guide to Intelligent Systems. Harlow: Pearson Education, 2005.

[16] M. S. A. Megat Ali, et al., "Classification of Kolb's Learning Styles using EEG Sub-Band Spectral Centroid Frequencies and Artificial Neural Network," Asian Journal of Scientific Research, vol. 9, no. 5, pp. 234-241, 2016.

[17] M. Y. Mashor, "Hybrid Multilayered Perceptron Networks," International Journal of Systems Science, vol. 31, no. 6, pp. $771-785,2000$.

[18] N. A. Mat Isa and W. M. F. W. Mamat, "Clustered-Hybrid Multilayer Perceptron Network for Pattern Recognition Application," Applied Soft Computing, vol. 11, pp. 1457-1466, 2011.

[19] A. L. Goldberger, et al., "PhysioBank, PhysioToolkit, and PhysioNet: Components of a New Research Resource for Complex Physiologic Signals," Circulation, vol. 101, no. 23, e215-e220, 2000.

[20] J. G. Proakis and D. G. Manolakis, Digital Signal Processing: Principles, Algorithms and Applications, 6th ed. New Jersey: Prentice Hall, 2006.

[21] M. S. A. Megat Ali, et al., "Detection of Cardiomyopathy using Multilayered Perceptron Network," in Proceedings of 2012 IEEE 8th International Colloquium on Signal Processing and its Applications, 2011, pp. 436-440.

[22] B. Younes, et al., "Lithium-Ion Batteries Modeling and State of Charge Estimation using Artificial Neural Network," International Journal of Electrical and Computer Engineering (IJECE), vol. 7, 2019.

[23] J. S. Smith, et al., "Neural Network Training With Levenberg-Marquardt and Adaptable Weight Compression," IEEE Transactions on Neural Networks and Learning Systems, vol. 30, pp. 580-587, 2019.

[24] P. Marzuki, et al., "A Design of License Plate Recognition System using Convolutional Neural Network," International Journal of Electrical and Computer Engineering (IJECE), vol. 9, 2019.

[25] A. I. Amiruddin, et al., "Feature reduction and arrhythmia classification via hybrid multilayered perceptron network," in Proceedings of 2013 IEEE 3rd International Conference on System Engineering and Technology, 2013, pp. 290-294. 
[26] Y. A. Lee, et al., "FPGA-based Architecture of Hybrid Multilayered Perceptron Neural Network," Indonesian Journal of Electrical Engineering and Computer Science (IJEECS), vol. 14, 2019.

\section{BIOGRAPHIES OF AUTHORS}
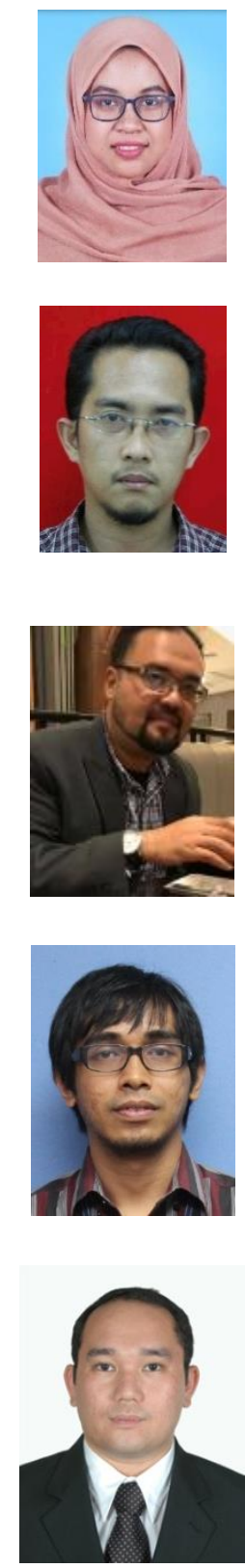

Fatin Syahirah Ab Gani is currently completing her final year studies in B. Eng (Electronics) at the Faculty of Electrical Engineering, Universiti Teknologi MARA, Malaysia. Her research interests are in biomedical signal processing and artificial intelligence.

Mohd Khairi Nordin received his Diploma in Electromechanical Engineering from Politeknik Ungku Omar, Malaysia, B.Eng. (Electrical) and M.Sc. in Electrical Engineering from Universiti Teknologi MARA, Malaysia. He is a lecturer at the Faculty of Electrical Engineering, Universiti Teknologi MARA. His research interests are in sensor and instrumentation, with application in agrotechnology.

Ahmad Ihsan Mohd Yassin received his B.Sc. in Electrical Engineering from Universiti Tun Hussein Onn, Malaysia, M.Sc. as well as Ph.D. in Electrical Engineering from Universiti Teknologi MARA, Malaysia. Dr. Ihsan is currently a senior lecturer at the Faculty of Electrical Engineering, Universiti Teknologi MARA. His research interests are in deep learning, blockchain technology, optimization, and system identification.

Idnin Pasya received his B.Eng. and M.Eng. in Information and Communication Engineering, as well as Ph.D. in Information, Communication and Media Design Engineering from Tokyo Denki University, Japan. He is a senior lecturer at the Faculty of Electrical Engineering, Universiti Teknologi MARA, Malaysia. Prior to joining the academia, Dr. Idnin has worked as an engineer at Toshiba PC \& Network, Tokyo, Japan. His research interests are in wideband communication systems, MIMO radar and its applications. He is currently the Deputy Director of Microwave Research Institute, Universiti Teknologi MARA.

Megat Syahirul Amin Megat Ali received his B.Eng. (Biomedical) from University of Malaya, Malaysia, M.Sc. in Biomedical Engineering from University of Surrey, United Kingdom, and Ph.D. in Electrical Engineering from Universiti Teknologi MARA, Malaysia. He is currently a senior lecturer at the Faculty of Electrical Engineering, Universiti Teknologi MARA. His research interests include biomedical signal processing and artificial intelligence. Dr. Megat is also a research fellow at the Microwave Research Institute, Universiti Teknologi MARA. 